\title{
Coarse-Fine Convolutional Deep-Learning Strategy for Human Activity Recognition
}

\author{
Carlos Avilés-Cruz *,+®i), Andrés Ferreyra-Ramírez ${ }^{\dagger}$ and Arturo Zúñiga-López ${ }^{\dagger}$ \\ and Juan Villegas-Cortéz ${ }^{+}($i)
}

Autonomous Metropolitan University. Electronics Department, Av. San Pablo 180, Col. Reynosa, C.P. 02200 Mexico City, Mexico; fra@azc.uam.mx (A.F.-R.); azl@azc.uam.mx (A.Z.-L.); juanvc@azc.uam.mx (J.V.-C.)

* Correspondence: caviles@azc.uam.mx; Tel.: +52-555-318-9030

+ These authors contributed equally to this work.

Received: 08 February 2019; Accepted: 24 March 2019; Published: 31 March 2019

\begin{abstract}
In the last decade, deep learning techniques have further improved human activity recognition (HAR) performance on several benchmark datasets. This paper presents a novel framework to classify and analyze human activities. A new convolutional neural network (CNN) strategy is applied to a single user movement recognition using a smartphone. Three parallel CNNs are used for local feature extraction, and latter they are fused in the classification task stage. The whole CNN scheme is based on a feature fusion of a fine-CNN, a medium-CNN, and a coarse-CNN. A tri-axial accelerometer and a tri-axial gyroscope sensor embedded in a smartphone are used to record the acceleration and angle signals. Six human activities successfully classified are walking, walking-upstairs, walking-downstairs, sitting, standing and laying. Performance evaluation is presented for the proposed CNN.
\end{abstract}

Keywords: CNN; deep-learning; classification; human action recognition

\section{Introduction}

Human Activity Recognition (HAR) is the automatic understanding of human actions performed by an individual or group of people. There are numerous areas and sectors where it is applied, such as smartphones, tablets, cars, games, health, security, commercial organizations and governments $[1,2]$. It is always been approached using sensors, namely using a video camera, infrared camera, microphone, GPS, gyroscope, accelerometer, proximity sensor, ultrasound sensor, light sensor, etc. [3-6]. Most of the sensors cited previously are integrated into a smartphone. On the other hand, in the recent years, smartphones have been preferred for implementing better HAR systems $[7,8]$ due to the increasing accuracy of their built-in sensors, popularity, low cost, wireless facilities, and wireless connectivity. Due to the aforementioned reasons, smartphones are opening a new horizon in the applications of understanding users' personal activities and their world contexts. In addition, the literature reports that the HAR systems embedded in smartphones are reaching good performance, however, they have not reached $100 \%$ recognition [3-6]. Single user activities that all HAR systems want to identify are walking, walking-upstairs, walking-downstairs, sitting, standing and laying, among others. The significance of the 6 activities identified in our proposed article is geared toward a future ability to assist people with disabilities in everyday household activities via a single smartphone, such as walking, ascending stairs, descending stairs, sitting, standing and laying. There are commercial HAR platforms developed by important companies such as google (https:/ / developers.google.com/ location-context/activity-recognition/), Microsoft-Azure (http:/ /www.md2c.nl/meetup-microsoftdata-science-azure-machine-learning-workshop/), and IBM-human action recognition (https:/ / www.ibm.com/blogs/research/category/ai/). Google "activity recognition" API identifies 2 types of 
movement within the 6 that we identify successfully, google HAR is interested in driving, walking, exercising, working, and playing activities. The API returns a value from 0 to 100 indicating the likelihood that the user is performing this activity. On the other hand, Microsoft-Azure API identifies sitting, standing up, standing, sitting down, walking activities, based on the use of wearable sensors. Azure API uses 4 wearing accelerometers from LiliPad Arduino positioned in the waist, left thigh, right ankle, and right arm. Azure API is not for generic use, compared to the sensors embedded in smartphones. Azure API identifies 3 types of movement within the 6 that we identify successfully. Finally, IBM-human action recognition API is oriented to HAR identification through visual information using cameras, which is beyond the scope of this article.

In this paper, a novel coarse-fine convolutional deep-learning strategy for human activity recognition is proposed which consists of three parallel CNNs that are fine-CNN, medium-CNN, and coarse-CNN. The outputs of the CNNs are flattened into a one-dimensional vector and used for the object's classification. In this proposal, a fully-connected layer is comprised of one input layer, one hidden layer, and one output layer. Concerning the CNNs components of the proposed architecture, the fine-CNN consists of four feature extraction (FE) layers where each FE layer performs convolution and max-pooling tasks. Referring to the medium-CNN, it contains two FE layers, convolution and max-pooling each. Finally, the coarse-CNN has only one FE layer, applying one convolution and one max-pooling task. For all CNNs, a ReLU activation function was applied.

Because HAR research has matured, there are several public benchmark human activity datasets [3,9-12] that allow a direct comparison of different activity recognition methods. In this work, the UCI HAR dataset [3] and the WISDM dataset [13] are selected which receive signals from an accelerometer and a gyroscope smartphone sensor. Dataset recordings have six human activities: walking, walking-upstairs, walking-downstairs, sitting, standing and laying which were successfully classified throughout our proposal.

The contribution of this work is threefold. Firstly, an improved convolutional neural network for human activity recognition is implemented which successfully merges coarse, medium, and fine features information. Secondly, the parallel feature extracted in the classification layer is carried out. Finally, an improvement in the classification accuracy using a coarse-fine deep-learning strategy when compared with the related HAR works is reported.

The rest of the paper is organized as follows. Section 2 discusses the state of the art. The proposed methodology is given in Section 3. Section 4 presents the system architecture. Experiments are discussed in Section 5, followed by a comprehensive discussion on evaluation in Sections 6 and 7 . Finally, Section 8 provides discussion, conclusions and briefly clarifies the possible future direction to advance the subject.

\section{State of the Art}

In the last decade, this research area has received significant attention due to the increasing trend of HAR applications in different areas, reduction in sensor price and built-in sensors in handheld devices. The human actions are identified by applying the extraction or selection features in the time or frequency domain on the signals detected by a smartphone's sensor. Since there are no working features that can ensure $100 \%$ identification of all the activities a person can perform, the problem still persists and requires further attention from the researchers. From sensor-based HAR research, there are two approaches.

- Video camera sensor: This research area is focused to identify HAR developed by a group of people. The most distinguished studies carried out for analyzing whole videos are [14-17]; 3D videos[18,19] or still images [16].

- Infrared camera, microphone, GPS, gyroscope, accelerometer, proximity sensor, ultra-sound sensor and light sensor: This HAR research is developed to identify a single person activity. There are survey works giving a landscape on different techniques and terminologies [14,20-23]. 
We are interested in this emerging research area which uses a smartphone sensor for single user recognition. The main works and their techniques are described as follows.

\subsection{Machine Learning-Based HAR Methods}

Focusing on the bibliography for a single person movement identification by a smartphone, the field of machine learning-based HAR methods reports a competitive work by Anguitia [3] which uses statistical features such as mean, minimum, maximum, standard deviation, skewness, kurtosis, angles, entropy, correlations, energy, and energy bands. The authors used support vector machine (SVM) as a classification system and they achieved good results to identify 6 human activities (walking, walking-upstairs, walking-downstairs, sitting, standing and running). The second most related work is "Human activity recognition by smartphone" by Le Tuan [4]. The author used time-domain and frequency-domain features: mean, minimum, maximum, standard deviation, energy, inter-quartile, entropy, auto-regression, correlations, skewness, kurtosis, the energy of a frequency; getting a 561-feature vector as an activity descriptor. The authors used a naive Bayes classifier and a Decision Tree criteria. Another important method is proposed in [5] were statistical features are used. By employing time-frequency features, the authors obtained good results to identify the same 6 human activities as two previous works. Other relevant work is based on Bag-of-Features [6] using a hierarchical recognition scheme over motion primitives, motion vocabulary size, weighting schemes of motion primitive assignments, and learning machine kernel functions. Also, Lane's work [24] used a Bayesian classifier to identify 4 to 6 human activities (walking, walking-upstairs, walking-downstairs, sitting, standing and running). Other researchers [25-27] used a $k$-nn classifier, Kim et al. used SVM [28], Quadratic Discriminant Analysis QDA [29], Multilayer Neural Network [30], Probabilistic Neural Network [31], and Classification Rules [32].

Finally, there are works where authors applied a Hidden Markov Model for segmenting human activities [33,34], using the same public database in [3], authors obtained good results to identify the same 6 human activities considered in this research work and, they defined an "Activity sequence modeling" to identify the relationship among activities.

\subsection{Convolutional Neural Network-Based HAR Methods}

A different approach to feature extraction task is based on deep learning/CNNs, and several works have been conducted to adapt it to the HAR problem. The most related work using CNNs is [35] where authors used the "divide and conquer" paradigm and 1D convolutional neural network to identify the actions performed by humans, six activities are efficiently identified: walk, walk upstairs, walk downstairs, sit, stand, and lay. Despite the good classification, the authors did not achieve $100 \%$ accuracy. Another close work by Ignatov [36] presents a user-independent deep learning-based approach for online human activity classification. Ignatov proposes to use Convolutional Neural Networks for local feature extraction together with simple statistical features that preserve information about the global form of time series. The author investigated the impact of time series length on the recognition accuracy and limited it up to one second that makes possible the continuous real-time activity classification. The accuracy of the proposed approach is evaluated on two commonly used WISDM and UCI datasets.

Other less accurate works in HAR, using CNNs as a platform base are [37-40]. There are approaches exploiting deep Recurrent Neural Network (RNN) [41] or combined Long Short-Term Memory (LSTM) RNN with CNN. Ordonez and Roggen [42] proposed DeepConvLSTM that combined convolutional and recurrent layers. Edel et al. [43] proposed a binarized bidirectional LSTM-RNNs which reduces memory consumption and replaced most of the arithmetic operations with bitwise operations achieving an increase in power-efficiency.

Despite the variety of proposals in the HAR field using convolutional or recurrent networks, there is still an opportunity for work to achieve $100 \%$ recognition of human activities. In this paper, a novel framework is proposed to analyze and classify single user activity using a smartphone on the 
well-known public smartphones databases [3,13]. Our scheme is based on a coarse-fine convolutional neural network strategy which is explained in the following section.

\section{Proposal}

The architecture of the proposed coarse-fine CNN system is shown in Figure 1. CNN is a parallel feedback neural network whose structure is inspired by the visual biological system. The main idea is the hierarchization of the information visually analyzed. On one side, the "coarse" information is perceived, i.e., circles, lines, shapes, and colors. On the other side, the "average" information, and finally, the "fine" detailed information is perceived. In the present proposal, detailed information is represented by several stages of convolution and max-pooling, while "coarse" information is represented by a single stage of convolution and pooling. The three levels of information are merged in the whole classification CNN stage. The overall structure of CNNs is described below:

- Convolutional layer: In one-dimensional case, a convolution between two vectors $x \in \mathbb{R}^{N}$ and a kernel vector $h \in \mathbb{R}^{M}$ is a vector $c \in \mathbb{R}^{M+N-1}$, where $c=x * h$, * represents the convolution operation. Thus, in discrete domain, the convolution is expressed as $c[n]=\sum_{k=-\infty}^{\infty} x[k] h[n-k]$, for $\forall n \in[1 \ldots N]$. In other words, a reflected vector $h$, which is also called a convolutional filter, is sliding along signal $x$, a dot product is computed at each $n$ value and the concatenated values $c[1], c[2], \ldots c[i]$ form the outputs of the convolutional layer $c[n]$.

- Activation function: Among the main non-linear activation functions such as sigmoidal, tangent, hyperbolic tangent, and ReLU, the latter is used in this proposal. Rectified linear unit (ReLU) is defined as $\operatorname{ReLU}(c)=\max (0, c)$. The effect produced by the ReLU function is thresholding of convolution $c$ with respect to zero value, obtaining, only positive values of $c$.

- Pooling layer: The aim of this stage is to reduce and summarize the convolutional output. Two typical pooling functions are used, the max pooling and mean pooling function. In this proposal, the max pooling function is used with a vector size of $[1 \times 2]$.

- Full-connected layer: This stage concatenates the outputs of the three partial CNNs: a fine-CNN, a medium-CNN, and a coarse-CNN. The output of the partial CNNs is flattened into a one-dimensional vector and used for the classification. In this proposal, a fully-connected layer is comprised of one input layer, one hidden layer, and one output layer.

- Soft-max layer: Finally, the output of the last layer is passed to a soft-max layer that computes the probability distribution over the predicted walking, up-stairs, down-stairs, sitting, standing and laying human activities.

All three partial CNNs: a fine-CNN, a medium-CNN, and a coarse-CNN are trained as a whole one. Training and optimizing tasks are carried out using a back propagation algorithm and stochastic gradient descent, respectively. 


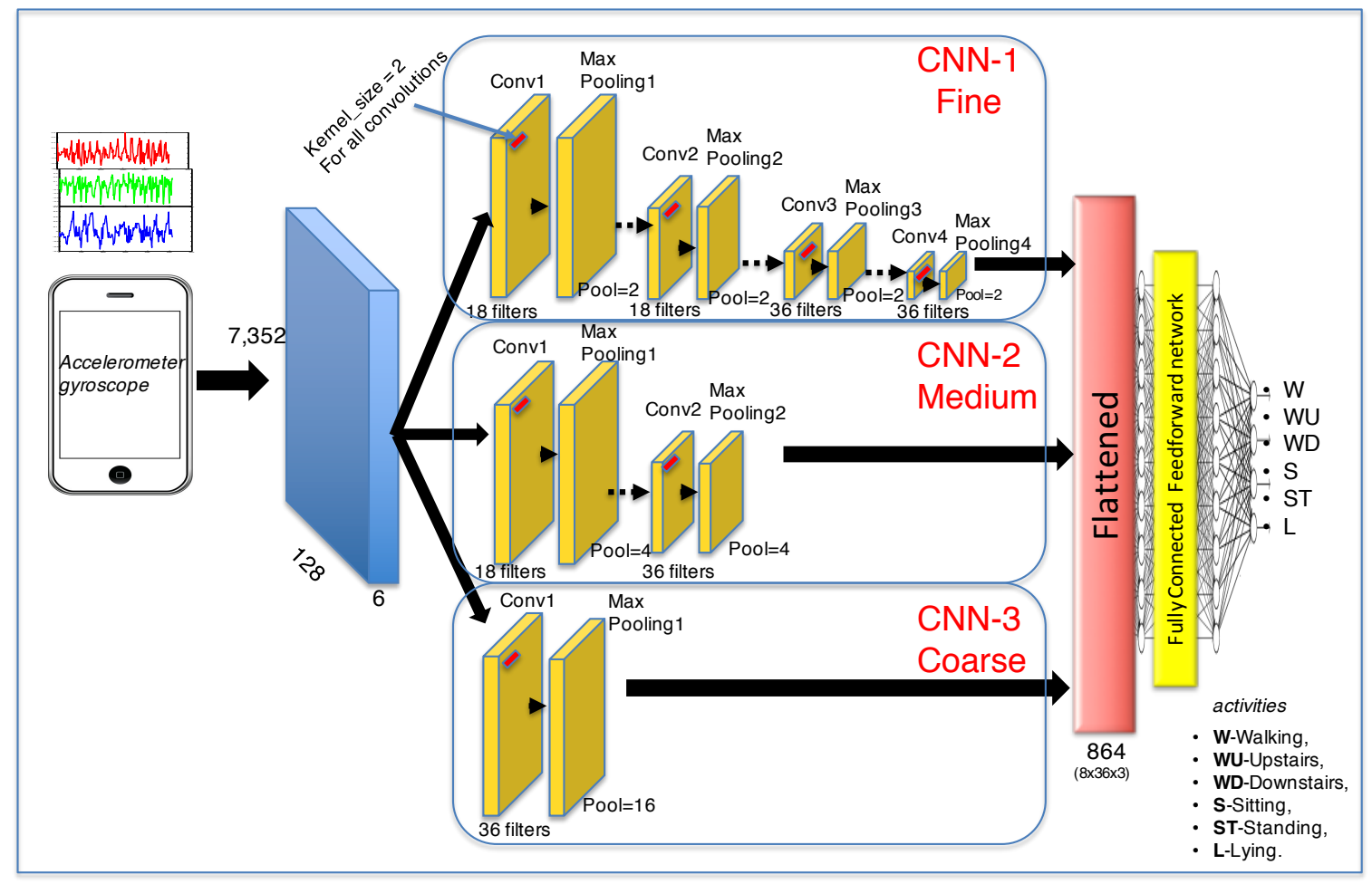

Figure 1. Proposed Coarse-fine convolutional neural network topology.

\section{System Architecture}

The whole proposed CNN architecture presented in Figure 1 is fed by six signals coming from an accelerometer and a gyroscope. The input data passes throughout the three partial CNN as follow:

- Fine-CNN (See Figure 2a): A first convolutional layer comprised of 18 filters where the kernel filter $h_{1}$ has the size $1 \times 2$ and the step of the convolution is 1 . Then, a max-pooling $g_{1}$ layer is applied with a size of $1 \times 2$ and the step of max-pooling $g_{1}$ is 2 . The activation function is ReLU. Then, a second convolutional layer, comprised of 18 filters where the kernel filter $h_{2}$ has the size $1 \times 2$ and the step of the convolution 2 is 1 . Then, a max-pooling $g_{2}$ layer is applied with a size of $1 \times 2$ and the step of max-pooling 2 is 2 . The activation function is ReLU. A third convolutional layer comprised of 36 filters where the kernel filter $h_{3}$ has the size $1 \times 2$ and the step of the convolution 3 is 1 . Then, a max-pooling 3 layer is applied with a size of $1 \times 2$ and the step of max-pooling $g_{3}$ is 2 . The activation function is ReLU. Finally, a fourth convolutional layer comprised of 36 filters where the kernel filter $h_{4}$ has the size $1 \times 2$ and the step of the convolution 4 is 1 . Then, a max-pooling 4 layer is applied with a size of $1 \times 2$ and the step of max-pooling 4 is 2 . The activation function is ReLU.

- Medium-CNN (See Figure $2 b$ ): For this CNN, first convolutional layer comprised of 18 filters where the kernel filter $h_{1}$ has the size $1 \times 2$ and the step of the convolution is 2 . Then, a max-pooling 1 layer is applied with a size of $1 \times 4$ and the step of max-pooling $g_{1}$ is 2 . The activation function is ReLU. Then, a second convolutional layer, comprised of 36 filters where the kernel filter $h_{2}$ has the size $1 \times 2$ and the step of the convolution 2 is 3 . Then, a max-pooling $g_{2}$ layer is applied with a size of $1 \times 4$ and the step of max-pooling 2 is 2 . The activation function is also ReLU.

- Coarse-CNN (See Figure 2c): For the last partial CNN, only one convolutional layer comprised of 36 filters where the kernel filter $h_{1}$ has the size $1 \times 2$ and the step of the convolution is 2 . Then, a max-pooling $g_{1}$ layer is applied with a size of $1 \times 16$ and the step of max-pooling $g_{1}$ is 2 . The activation function is ReLU. 


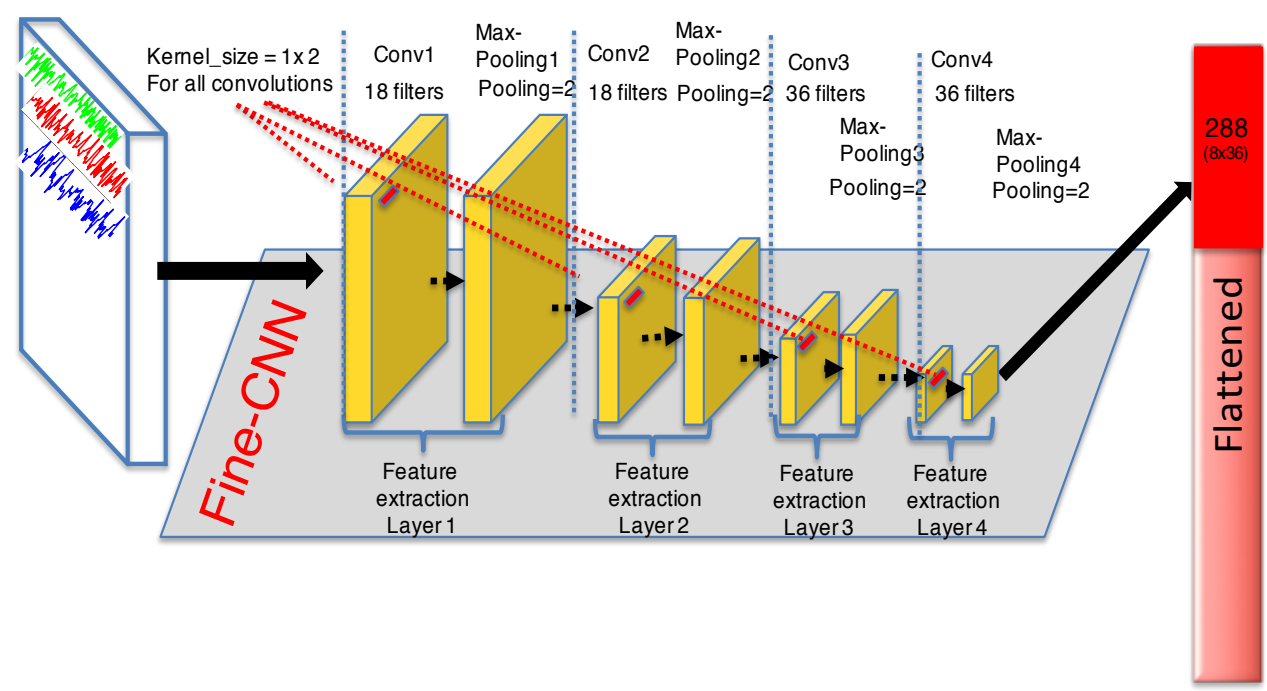

(a) Architecture of a fine-CNN

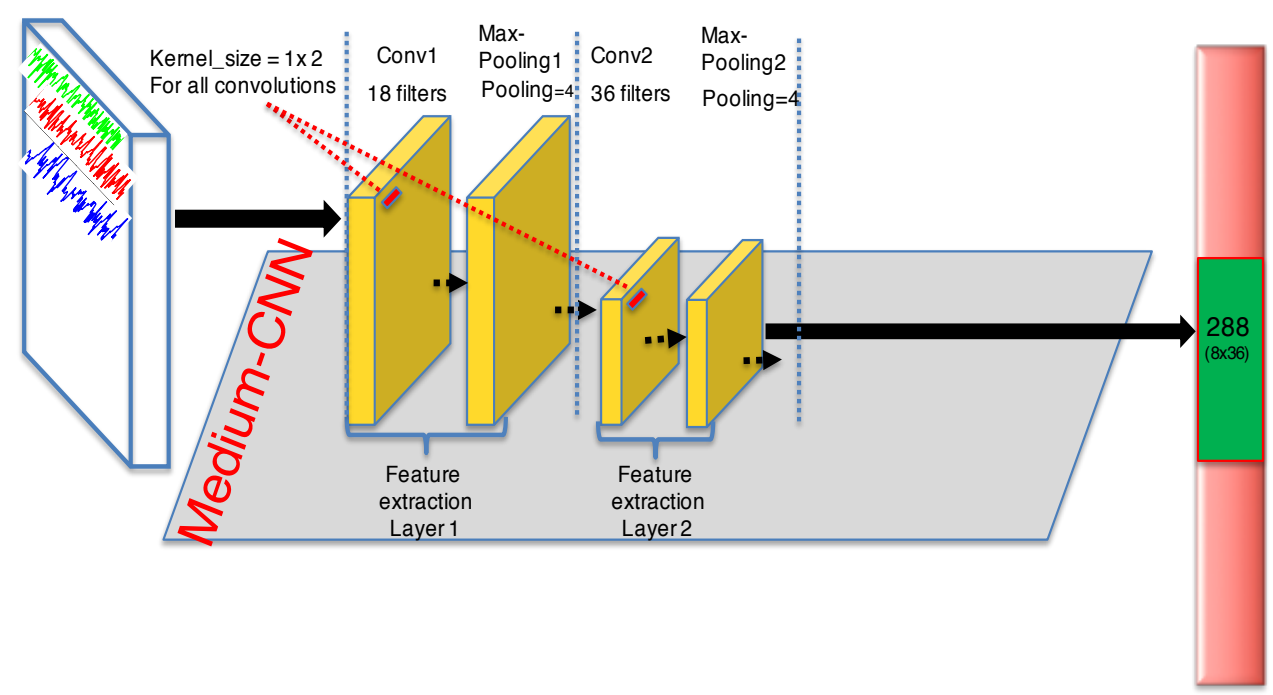

(b) Architecture of a medium-CNN

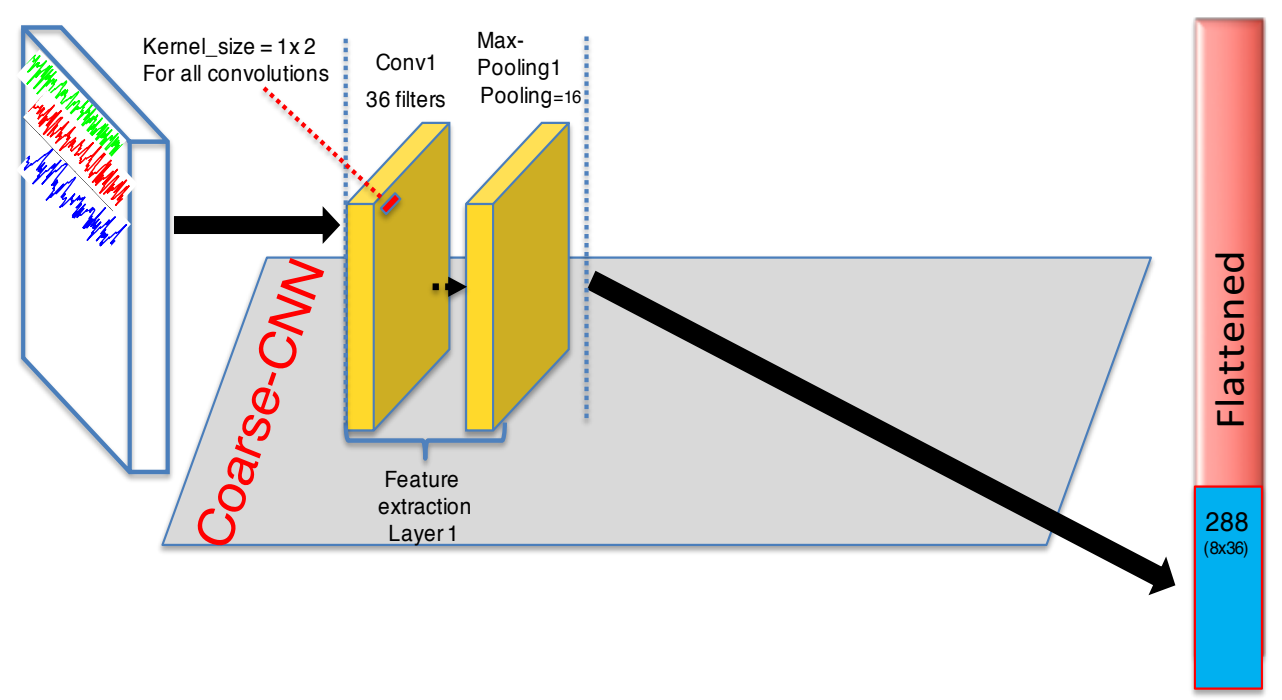

(c) Architecture of a coarse-CNN

Figure 2. Partial CNN proposed architecture. 
The output of the three partial max-pooling output layers are then flattened. The joint vector is subsequently passed to a fully-connected layer that consists of 864 neurons $(8 \times 36 \times 3)$. We have used a dropout technique in this layer with dropout rate of 0.00005 . Finally, the outputs of the fully-connected layer are passed to a soft-max layer that computes probability distribution over six activity classes. The model is trained to minimize cross-entropy loss function using back propagation algorithm and optimize training parameters with stochastic gradient descent [44].

For the proposed fine-coarse $\mathrm{CNN}$, the loss entropy function is defined as:

$$
\zeta_{T}\left(\Theta_{1}, \Theta_{2}, \Theta_{3}, W\right)=\zeta_{F M C}\left(\Theta_{1}, \Theta_{2}, \Theta_{3}\right)+\zeta_{C L A}(W)
$$

where $\zeta_{F M C}\left(\Theta_{1}, \Theta_{2}, \Theta_{3}\right)$ corresponds to the loss function of fine-CNN, medium-CNN, and coarse-CNN; and $\zeta_{C L A}(W)$ corresponds to the loss function of the whole classification layer (dropout and soft-max layers). Total loss function $\zeta_{T}$ can be rewritten as:

$$
\begin{aligned}
\zeta_{T}\left(\Theta_{1}, \Theta_{2}, \Theta_{3}, W\right)= & -\sum_{i=1}^{3} \log \left[\hat{p}\left(m_{h} / \Theta_{i}\right)\right]-\log \left[\hat{p}\left(m_{h} / W\right)\right] \\
=- & \log \left[\hat{p}\left(m_{h} / \Theta_{1}\right)\right]-\log \left[\hat{p}\left(m_{h} / \Theta_{2}\right)\right]- \\
& \log \left[\hat{p}\left(m_{h} / \Theta_{3}\right)-\log \left[\hat{p}\left(m_{h} / W\right)\right]\right.
\end{aligned}
$$

where $\Omega=\left\{\Theta_{1}, \Theta_{2}, \Theta_{3}\right\}$ are the parameter sets for the three partial CNN. $W$ is the parameter sets for the classification layer (dropout and soft-max layers). $m_{h}=$ movement type, where $h=$ \{walking, walking-upstairs, walking-downstairs, sitting, standing and laying\}. $\hat{p}\left(m_{h} / \Theta_{i}\right)$ stands for the conditional probability function for a given movement type conditioned to a $\Theta_{i}$ parameter sets, and $\hat{p}\left(m_{h} / W\right)$ stands for the conditional probability function for a given movement type conditioned to a $W$ classification parameter sets layer.

The parameter set for each partial CNN is defined as follows:

$$
\begin{aligned}
& \Theta_{1}=\text { parameter sets for the fine-CNN } \\
& \Theta_{2}=\text { parameter sets for the medium-CNN } \\
& \Theta_{3}=\text { parameter sets for the coarse-CNN } \\
& W=\text { parameter sets for the classification layer (dropout and soft-max layers) }
\end{aligned}
$$

\section{Experiments}

\subsection{UCI HAR Dataset}

Accelerometer and gyroscope sensors built-in in a smartphone were used to collect two-tri-axial movement information [3]. Sensor's data were collected from 30 volunteers, between the age of 19-49 year. Carrying a smartphone Samsung Galaxy SII in a vertical position in their pockets, each subject performed six activities: walking, walking up stairs, walking down stairs, sitting, standing, and laying activity. 3-axial linear acceleration and 3-axial angular velocity data were collected. These sensor's data were sampled at a constant rate of $50 \mathrm{~Hz}$, using the embedded accelerometer and gyroscope. A realization of a single activity was divided into windows of $2.56 \mathrm{~s}$ each, which is sampled at $50 \mathrm{~Hz}$ giving 128 samples $(2.56 \mathrm{~s} \times 50 \mathrm{~Hz}=128)$. The database is structured into two sets, $70 \%$ of the volunteers ( 21 persons) were selected for training and 30\% for testing (9 persons). Table 1 shows the activities distribution over the two sets. An example of a single recording can be found in Figure 3 where four activities are shown. Table 2 shows the hyperparameters experimental setup. As you can see in pooling size parameter, there are three vector size $[1 \times 2],[1 \times 4]$ and $[1 \times 16]$ for fine-CNN, medium-CNN and coarse-CNN, respectively (See Figure 2). 


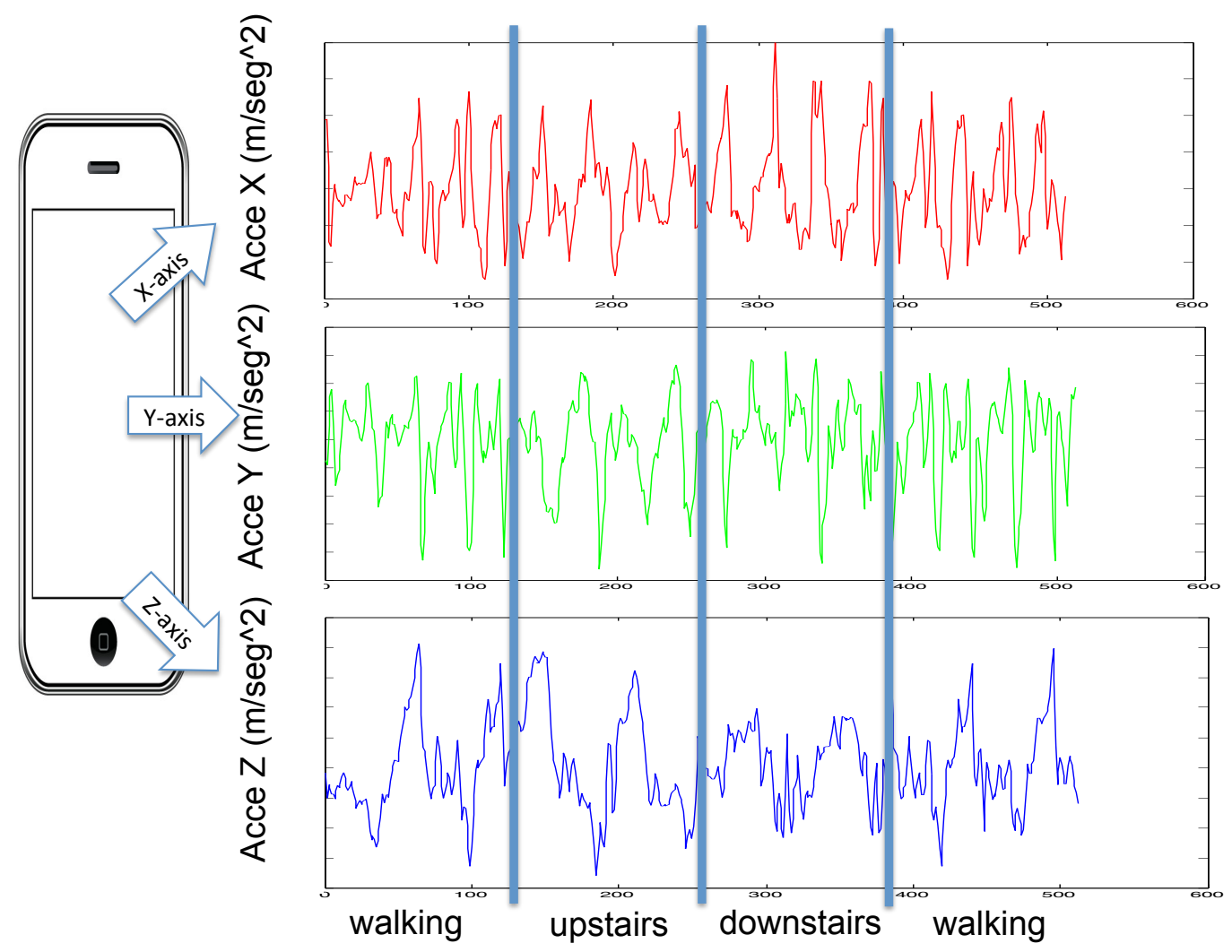

Figure 3. Example of four human activities: walking, walking-upstairs, and walking-downstairs.

Table 1. Activities distribution over the training and testing sets in UCI HAR database.

\begin{tabular}{lcc}
\hline & Training & Testing \\
\hline walking & 1226 & 496 \\
walking-upstairs & 1073 & 471 \\
walking-downstairs & 986 & 420 \\
sitting & 1286 & 491 \\
standing & 1374 & 532 \\
laying & 1407 & 537 \\
\hline Total & 7352 & 2947 \\
\hline
\end{tabular}

Table 2. Experiment setup.

\begin{tabular}{ll}
\hline Parameter & Value \\
\hline The size of input vector & 128 \\
The number of input channels & 6 \\
Filter size & {$[1 \times 2]$} \\
Pooling size & {$[1 \times 2],[1 \times 4]$ or $[1 \times 16]$} \\
Activation function & ReLU (rectified linear unit) \\
Learning rate & 0.0001 \\
Weight decay & 0.00005 \\
Momentum & $0.5-0.99$ \\
The probability of dropout & 0.8 \\
The size of minibatches & 500 \\
Maximum epochs & 2000 \\
\hline
\end{tabular}




\subsection{Data Set}

\section{Subject-Dependent dataset}

For the training stage (See second column of Table 1), a full learning database was formed by 7352 trails of 21 volunteers ( $70 \%$ of the whole database). Training dataset is conformed of 7352 trials multiply by 128 samples multiply by 6 axis $(7352 \times 128 \times 6$ matrix $)$.

\section{Subject-Independent dataset}

For the testing stage (See third column of Table 1), a database was formed by 2947 trails of 9 volunteers ( $30 \%$ of the whole database). Testing dataset is conformed of 2947 trials multiply by 128 samples multiply by 6 axis $(2947 \times 128 \times 6$ matrix $)$.

All signals were filtered with a digital FIR low-pass filter with a cut-off frequency of $10 \mathrm{~Hz}$. Thus, the already filtered signals are used in the proposed neural network.

\section{Evaluation}

We implemented the proposed coarse-fine convolutional deep-learning strategy for human activity recognition on the python+Tensor Flow (python $=2.7$, tensorflow $=1.1$ ) platform running on iMac-XOS Intel Core i5 CPU. To evaluate the proposal, firstly, the influence of each partial CNN is evaluated, and then, whole parallel CNN strategy is evaluated. Performance evaluation for the three proposed CNN: Fine-CNN, Medium-CNN, and Coarse-CNN, as well as, for the proposed merged architecture is presented as follows.

\subsection{Learning Evaluation}

Evaluation is developed in training and testing tasks, regarding training task, accuracy and loss parameters are evaluated for the four CNNs. One of the most important parameter to be defined is the size of the convolutional filters, which was defined experimentally to $[1 \times 2]$. Figure 4 shows the classification accuracy curve, the Coarse-Fine $\mathrm{CNN}$ is not very sensitive to this parameter: while the first best accuracy was obtained for filter of size $[1 \times 2]$, the accuracy does not drop significantly till this size becomes greater than $[1 \times 2]$ size.

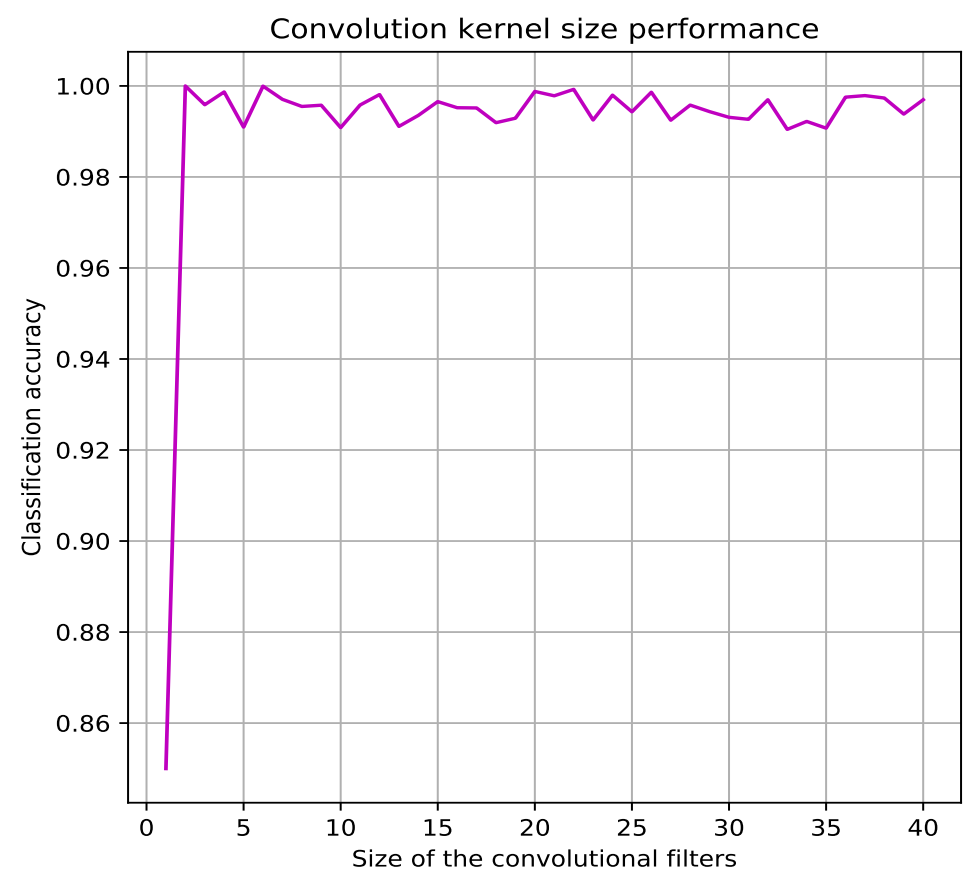

Figure 4. Dependency between the size of the convolutional filters and CNN accuracy. 
Figure 5 shows the first 500 iterations. In Figure 5 a the loss function performance for the four CNN proposed: Fine-CNN, Medium-CNN, Coarse-CNN and Proposed-CNN is shown. The proposed $\mathrm{CNN}$ (magenta plot) reaches minimum values in less iterations and obtains zero value at the end of the training task. Besides, Figure $5 \mathrm{~b}$ depicts the precision performance for the same four $\mathrm{CNN}$, it can be seen that it is the best performance reached by the proposed CNN (magenta plot), i.e., less iterations and best accuracy.

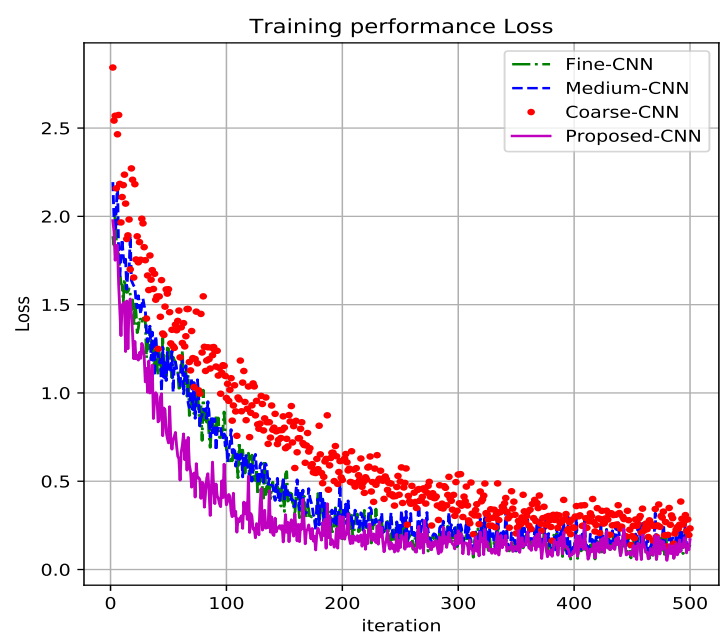

(a) Training loss

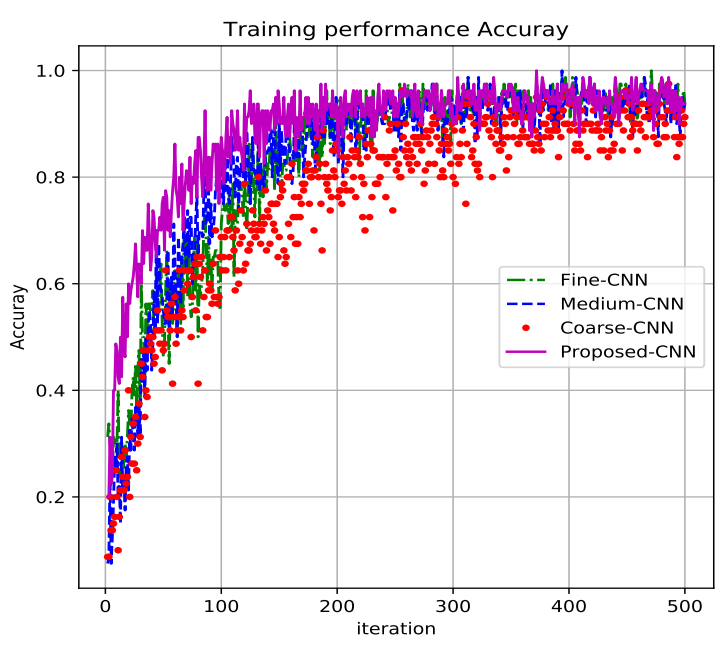

(b) Training accuray

Figure 5. Training results for the proposed CNN architecture.

Other parameters analyzed in the training task were training-validation loss and training-validation accuracy. Figure 6 shows the evolution curves through iterations. The training task is developed following the paradigm "Subject-dependent test", it means the same dataset is used for learning and testing task. From Figure 6, it can be seen that continued magenta line plot corresponds to the proposed $\mathrm{CNN}$ architecture where the accuracy reaches $100 \%$ (see Figure $6 \mathrm{~b}$ ), and the loss parameter reaches the zero value (see Figure 6a). Other color plots correspond to the partial CNN used and fused: red $\rightarrow$ coarse-CNN, blue $\rightarrow$ medium-CNN, and green $\rightarrow$ fine-CNN.

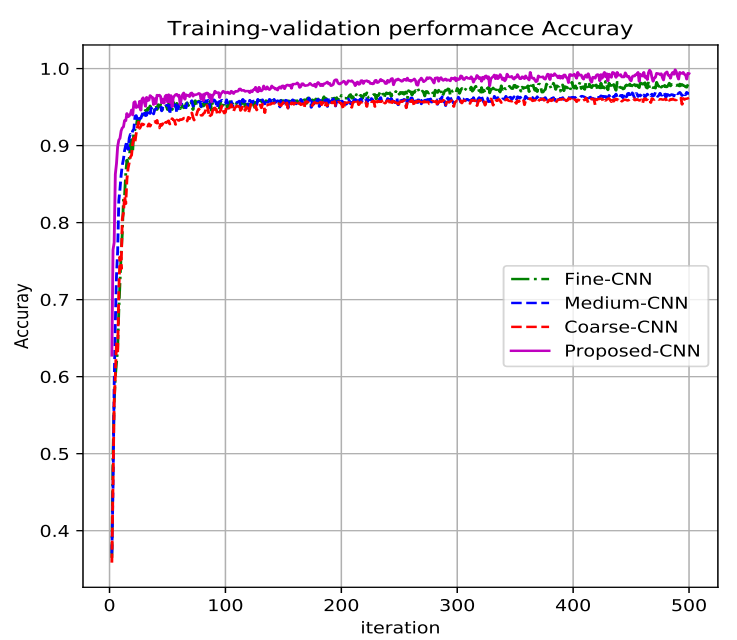

(a) Training-validation loss

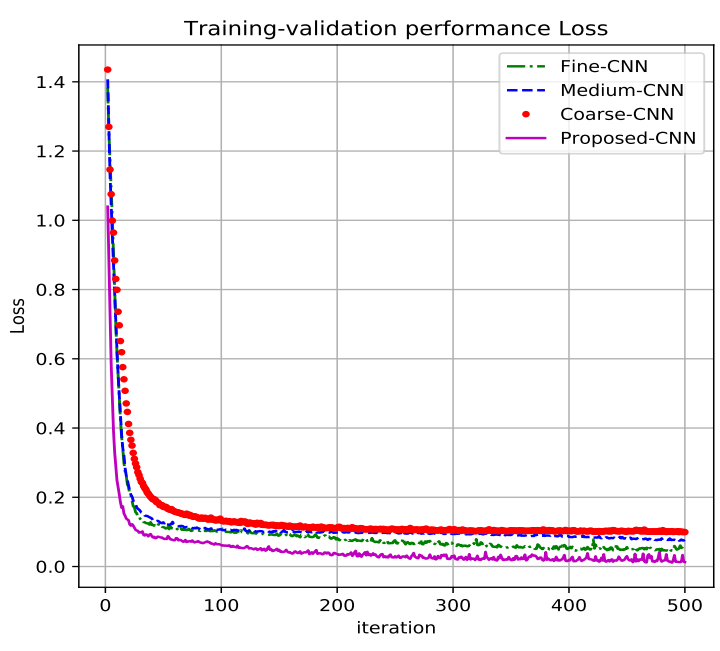

(b) Training-validation accuray

Figure 6. Training-validation results for the proposed $\mathrm{CNN}$ architecture. 


\subsection{Cross-Dataset Evaluation}

Figure 7 shows the testing performance for the proposed CNN. For this test, the testing dataset is completely different from the learning task. The test is developed following the paradigm "Subject-Independent test". From Figure 7, it can be seen that the continued magenta line plot corresponds to the proposed CNN architecture where the accuracy reaches $100 \%$. It seems that the fusion of partial information given by fine-CNN, medium-CNN, and coarse-CNN makes it possible to obtain a $100 \%$ of good classification for HAR activities.

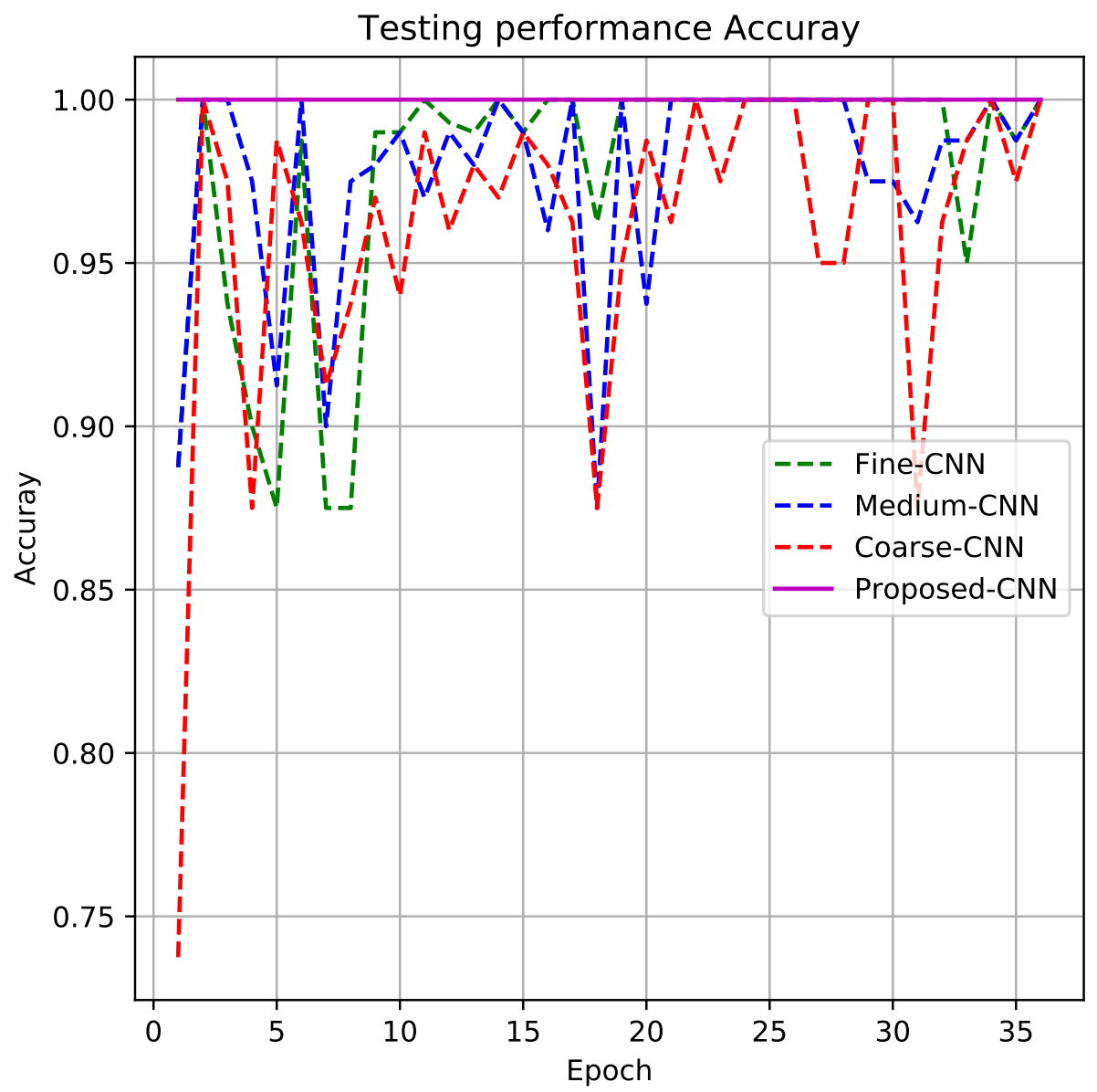

Figure 7. Testing results for the proposed CNN architecture.

Confusion matrix of the six single user activities classification in testing task is given in Table 3, performance activities are: walking activity $100 \%$, ascending stairs $100 \%$, descending stairs $100 \%$, sitting $100 \%$, standing $100 \%$ and laying $100 \%$; giving a mean average of $100 \%$.

Table 3. Testing confusion matrix of the six single user activities.

\begin{tabular}{|c|c|c|c|c|c|c|}
\hline & Walking & Ascending Stairs & Descending Stairs & Sitting & Standing & Laying \\
\hline walking & 100 & 0 & 0 & 0 & 0 & 0 \\
\hline ascending stairs & 0 & 100 & 0 & 0 & 0 & 0 \\
\hline descending stairs & 0 & 0 & 100 & 0 & 0 & 0 \\
\hline sitting & 0 & 0 & 0 & 100 & 0 & 0 \\
\hline standing & 0 & 0 & 0 & 0 & 100 & 0 \\
\hline laying & 0 & 0 & 0 & 0 & 0 & 100 \\
\hline
\end{tabular}




\subsection{Comparison to Related Work}

Table 4 and Figure 8 compare our coarse-fine convolutional deep-learning strategy for human activity recognition with the best competitive works reported in the literature. Please note that in this comparison, we present the classification performance for the six single user activities, as well as, the mean average performance. The comparison includes the two best competitive methods using machine learning approach $[3,33]$, and, on the other hand, the 3 most competitive works using convolutional networks [35,36,38]. As it can be seen, our proposal accurately recognizes $(100 \%)$ each of the movements under the scheme of fusion of fine, medium and coarse information from the defined convolutional neural networks.

As given in Table 4 where authors used the same database, the proposed method improves the best result from the literature about $2 \%$ i.e., from $98 \%$ San-Segundo [33] to $100 \%$.

Table 4. Comparison performance of the most competitive methods in detection of human motion activities recognition using the same database.

\begin{tabular}{lccccccc}
\hline & Walking & $\begin{array}{c}\text { Ascending } \\
\text { Stairs }\end{array}$ & $\begin{array}{c}\text { Descending } \\
\text { Stairs }\end{array}$ & Sitting & Standing & Laying & $\begin{array}{c}\text { Mean } \\
\text { Average }\end{array}$ \\
\hline Our proposal & $\mathbf{1 0 0} \%$ & $\mathbf{1 0 0} \%$ & $\mathbf{1 0 0} \%$ & $\mathbf{1 0 0} \%$ & $\mathbf{1 0 0} \%$ & $\mathbf{1 0 0} \%$ & $\mathbf{1 0 0} \%$ \\
San-Segundo [33] & $100 \%$ & $99 \%$ & $99 \%$ & $92 \%$ & $98 \%$ & $100 \%$ & $98 \%$ \\
Ignatov [36] & $99.4 \%$ & $100 \%$ & $98.81 \%$ & $90.04 \%$ & $98.2 \%$ & $100 \%$ & $97.63 \%$ \\
Cho [35] & $98.9 \%$ & $98.51 \%$ & $98.57 \%$ & $92.46 \%$ & $97.37 \%$ & $99.81 \%$ & $97.62 \%$ \\
Anguita [3] & $99 \%$ & $96 \%$ & $98 \%$ & $88 \%$ & $97 \%$ & 100 & $96 \%$ \\
Ronao [38] & $100 \%$ & $99.16 \%$ & $99.53 \%$ & $86.68 \%$ & $89.69 \%$ & 93.64 & $94.79 \%$ \\
\hline
\end{tabular}

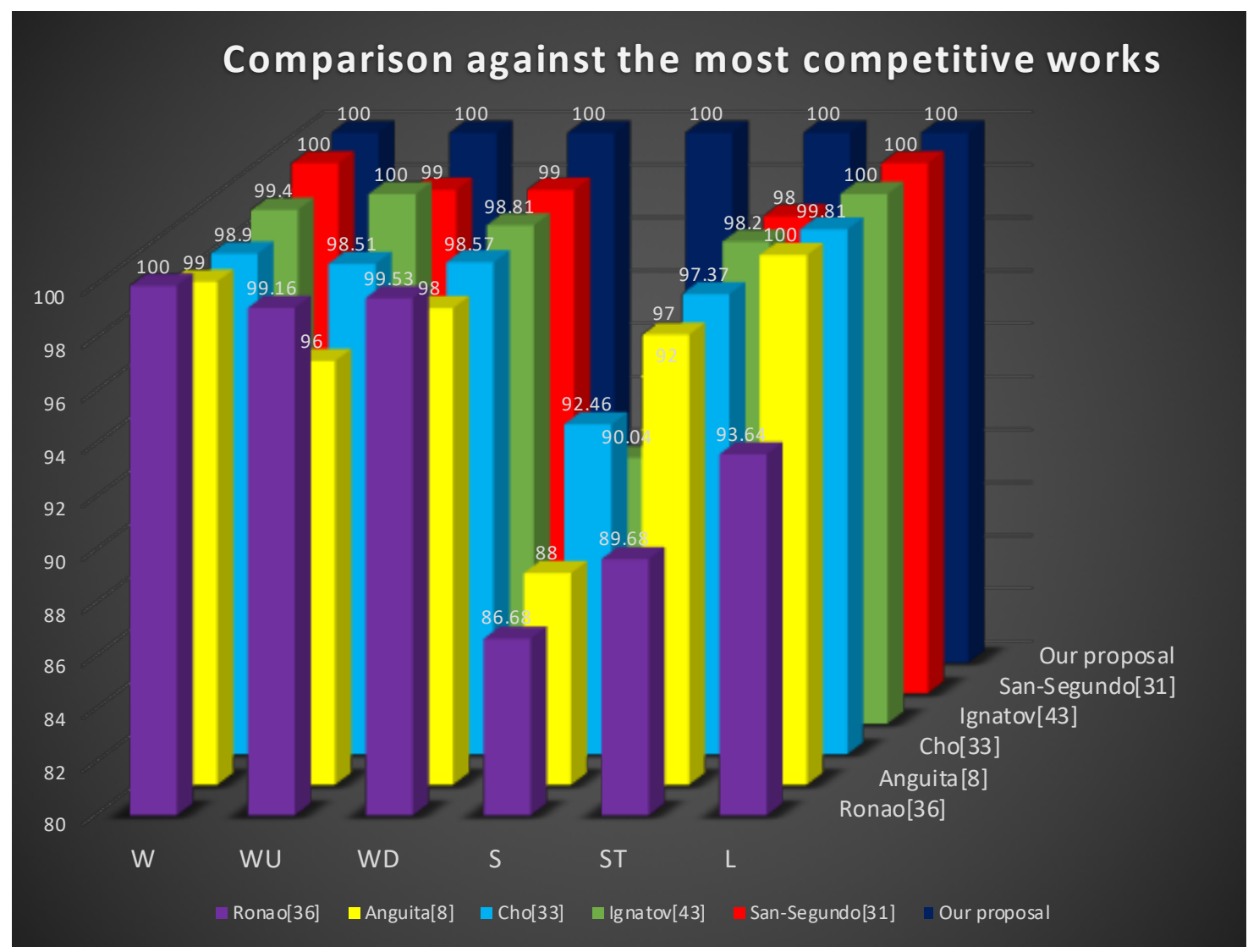

Figure 8. Performance comparison against the most competitive methods: W-> walking, WU->walking-upstairs, WD->walking-downstairs, S->sitting, ST->standing and L->laying. 


\section{WISDM Dataset}

To test our proposal, this paper, uses a second standard HAR dataset which is publicly available from the WISDM group [13]. The dataset is conformed of 6 activities walking, jogging, walking upstairs, walking downstairs, sitting, and standing. While performing these activities, the sampling rate for accelerometer sensor was set to $20 \mathrm{~Hz}$. Dataset description is shown in Table 5

Table 5. WISDM dataset description [13].

\begin{tabular}{ccc}
\hline Activity & Number of Samples & Percentage \\
\hline Walking & 424,400 & $38.6 \%$ \\
Jogging & 342,177 & $31.2 \%$ \\
Upstairs & 122,869 & $11.2 \%$ \\
Downstairs & 100,427 & $9.1 \%$ \\
Sitting & 59,939 & $5.5 \%$ \\
Standing & 48,397 & $4.4 \%$ \\
\hline
\end{tabular}

\subsection{Evaluation}

Following the same methodology of the proposed Coarse-fine convolutional network and with the same parameters defined in Section 5 "Experiments", the experimentation was carried out with the second WISDM dataset. The results obtained are shown in the confusion matrix where six single user activities classification in testing task is given in Table 6, performance activities are: walking activity $100 \%$, jogging $100 \%$, upstairs $100 \%$, downstairs $100 \%$, sitting $100 \%$ and standing $100 \%$; giving a mean average of $100 \%$.

Table 6. Testing confusion matrix of the six single user activities.

\begin{tabular}{ccccccc}
\hline & Walking & Jogging & Upstairs & Downstairs & Sitting & Standing \\
\hline walking & 100 & 0 & 0 & 0 & 0 & 0 \\
jogging & 0 & 100 & 0 & 0 & 0 & 0 \\
upstairs & 0 & 0 & 100 & 0 & 0 & 0 \\
downstairs & 0 & 0 & 0 & 100 & 0 & 0 \\
sitting & 0 & 0 & 0 & 0 & 100 & 0 \\
standing & 0 & 0 & 0 & 0 & 0 & 100 \\
\hline
\end{tabular}

\subsection{Comparison to Related Work}

Table 7 compares our coarse-fine convolutional deep-learning strategy for human activity recognition with the best competitive work reported in the literature. Please note that in this comparison, we present the classification performance for the six single user activities, as well as, the mean average performance. The comparison is versus the most competitive work using convolutional networks [45]. As it can be seen, our proposal accurately recognizes (100\%) each of the movements under the scheme of fusion of fine, medium and coarse information from the defined convolutional neural networks.

As given in Table 7 where the author used the same database and a CNN, the proposed method improves about $0.7 \%$ i.e., from $99.33 \%$ to $100 \%$.

Table 7. Comparison performance of the most competitive method in detection of human motion activities recognition using WISDM dataset.

\begin{tabular}{lccccccc}
\hline & Walking & Jogging & Upstairs & Downstairs & Sitting & Standing & Mean Average \\
\hline Our proposal & $\mathbf{1 0 0} \%$ & $\mathbf{1 0 0} \%$ & $\mathbf{1 0 0} \%$ & $\mathbf{1 0 0} \%$ & $\mathbf{1 0 0} \%$ & $\mathbf{1 0 0} \%$ & $\mathbf{1 0 0} \%$ \\
Shakya [45] & $98 \%$ & $99 \%$ & $100 \%$ & $99 \%$ & $100 \%$ & $100 \%$ & $99.3 \%$ \\
\hline
\end{tabular}




\section{Conclusions}

Human activity recognition is a challenging problem. In this paper, a novel CNN framework is presented to classify single user activities based on local feature extraction under parallel scheme. The whole CNN strategy is based on coarse-medium-fine feature extraction and then, their fusion in a classification stage.

The sensors used to record the acceleration and angle signals were a tri-axial accelerometer and a tri-axial gyroscope embedded in a smartphone.

Six human activities were successfully classified: walking, walking-upstairs, walking-downstairs, sitting, standing and laying, giving an average recognition of $100 \%$.

The experimental results show that our proposal outperforms the most competitive methods reported in San-Segundo [33], Ignatov [36], Cho [35], Anguita D. [3], Ronao [38], and [45]; with an average recognition of $100 \%$ for the same human activity.

Future work includes taking into account the more complex human activities and to find association relationships to health issues for common physical diseases.

Author Contributions: Writing-review and editing, C.A.-C.; investigation, C.A.-C., A.Z.-L. and A.F.-R.; resources, J.V.-C.; writing-original draft preparation, C.A.-C.; validation, C.A.-C., A.Z.-L. and A.F.-R.; conceptualization, C.A.-C.; formal analysis, C.A.-C., A.F.-R., A.Z.-L. and J.V.-C.; methodology, C.A.-C., and A.F.-R.; C.A.-C. supervised the overall research work. All authors contributed to the discussion and conclusion of this research.

Funding: This research received no external funding

Conflicts of Interest: The authors declare that there is no conflict of interests regarding the publication of this paper.

\section{References}

1. Lara, O.; Labrador, M. A survey on human activity recognition using wearable sensors. IEEE Commun. Surv. Tutor. 2013, 15, 1192-1209. [CrossRef]

2. Chen, L.; Hoey, J.; Nugent, C.; Cook, D.; Yu, Z. Sensor-based activity recognition. IEEE Trans. Syst. Man Cybern. Part C Appl. Rev. 2012, 42, 790-808. [CrossRef]

3. Anguita, D.; Ghio, A.; Oneto, L.; Parra, X.; Reyes-Ortiz, J.L. A Public Domain Dataset for Human Activity Recognition Using Smartphones. In Proceedings of the European Symposium on Artificial Neural Networks, Computational Intelligence and Machine Learning (ESANN 2013), Bruges, Belgium, 24-26 April 2013; ISBN 978-2-87419-081-0.

4. Le, T.D.; Nguyen, C.V. Human Activity Recognition by smartphone. In Proceedings of the 2nd National Foundation for Science and Technology Development Conference on Information and Computer Science, Ho Chi Minh City, Vietnam, 16-18 September 2015; pp. 219-224.

5. Liang, Y.; Zhou, X.; YU, Z.; Guo, B. Energy-Efficient Motion Related Activity Recognition on Mobile Devices for Pervasive Healthcare. Mob. Netw. Appl. 2014, 19, 303-317. [CrossRef]

6. Zhang, M.; Sawchuk, A.A. Motion Primitive-based Human Activity Recognition Using a Bag-of-features Approach. In Proceedings of the 2nd ACM SIGHIT International Health Informatics Symposium, Miami, Florida, USA, 28-30 January 2012; pp. 631-640.

7. Lane, N.; Miluzzo, E.; Lu, H.; Peebles, D.; Choudhury, T.; Cambell, A. A Survey of Mobile Phone Sensing. IEEE Commun. Mag. 2010, 48, 140-150. [CrossRef]

8. Liu, J.; Wang, Z.; Zhong, L.; Wickramasuriya, J.; Vasudevan, V. Wave: Accelerometer-Based Personalized Gesture Recognition and Its Applications. In Proceedings of the Seventh Annual IEEE International Conference on Pervasive Computing and Communications (PerCom 2009), Galveston, TX, USA, 9-13 March 2009.

9. Zappi, P.; Lombriser, C.; Stiefmeier, T.; Farella, E.; Roggen, D.; Benini, L.; Tröster, G. Activity Recognition from On-Body Sensors: Accuracy-Power Trade-Off by Dynamic Sensor Selection. In Proceedings of the 5th European Conference on Wireless Sensor Networks, Bologna, Italy, 30 January-1 February 2008; Verdone, R., Ed.; Springer: Berlin/Heidelberg, Germany, 2008; pp. 17-33. 
10. Roggen, D.; Calatroni, A.; Rossi, M.; Holleczek, T.; Forster, K.; Troster, G.; Lukowicz, P.; Bannach, D.; Pirkl, G.; Ferscha, A.; et al. Collecting complex activity datasets in highly rich networked sensor environments. In Proceedings of the 2010 Seventh International Conference on Networked Sensing Systems (INSS), Kassel, Germany, 15-18 June 2010; pp. 233-240.

11. Lockhart, J.W.; Weiss, G.M.; Xue, J.C.; Gallagher, S.T.; Grosner, A.B.; Pulickal, T.T. Design Considerations for the WISDM Smart Phone-based Sensor Mining Architecture. In Proceedings of the Fifth International Workshop on Knowledge Discovery from Sensor Data (SensorKDD '11), San Diego, CA, USA, 21 August 2011; ACM: New York, NY, USA, 2011; pp. 25-33.

12. Micucci, D.; Mobilio, M.; Napoletano, P. UniMiB SHAR: A new dataset for human activity recognition using acceleration data from smartphones. Appl. Sci. 2017, 7, 1101. [CrossRef]

13. Kwapisz, J.R.; Weiss, G.M.; Moore, S.A. Activity Recognition using Cell Phone Accelerometers. In Proceedings of the Fourth International Workshop on Knowledge Discovery from Sensor Data (at KDD-10), Washington, DC, USA, 25-28 July 2010.

14. Zhang, C.; Yang, X.; Lin, W.; Zhu, J. Recognizing Human Group Behaviors with Multi-group Causalities. In Proceedings of the The 2012 IEEE/WIC/ACM International Joint Conferences on Web Intelligence and Intelligent Agent Technology—Volume 03; IEEE Computer Society: Washington, DC, USA, 2012; pp. 44-48.

15. Fan, Y.; Yang, H.; Zheng, S.; Su, H.; Wu, S. Video Sensor-Based Complex Scene Analysis with Granger Causality. Sensor 2013, 13, 13685-13707. [CrossRef]

16. Zhou, Z.; Li, K.; He, X. Recognizing Human Activity in Still Images by Integrating Group-Based Contextual Cues. In Proceedings of the 23rd ACM International Conference on Multimedia (MM'15), Brisbane, Australia, 26-30 October 2015; ACM: New York, NY, USA, 2015; pp. 1135-1138.

17. Onofri, L.; Soda, P.; Pechenizkiy, M.; Iannello, G. A Survey on Using Domain and Contextual Knowledge for Human Activity Recognition in Video Streams. Expert Syst. Appl. 2016, 63, 97-111. [CrossRef]

18. Zheng, Y.; Yao, H.; Sun, X.; Zhao, S.; Porikli, F. Distinctive action sketch for human action recognition. Signal Process. 2018, 144, 323-332. [CrossRef]

19. Ji, X.; Cheng, J.; Feng, W.; Tao, D. Skeleton embedded motion body partition for human action recognition using depth sequences. Signal Process. 2018, 143, 56-68. [CrossRef]

20. Ghosh, A.; Riccardi, G. Recognizing Human Activities from Smartphone Sensor Signals. In Proceedings of the 22Nd ACM International Conference on Multimedia (MM'14), Multimedia Orlando, Fl, USA, 3-7 November 2014; ACM: New York, NY, USA, 2014; pp. 865-868.

21. Dao, M.S.; Nguyen-Gia, T.A.; Mai, V.C. Daily Human Activities Recognition Using Heterogeneous Sensors from Smartphones. Procedia Comput. Sci. 2017, 111, 323-328. [CrossRef]

22. Hui, S.; Zhongmin, W. Compressed sensing method for human activity recognition using tri-axis accelerometer on mobile phone. J. China Univ. Posts Telecommun. 2017, 24, 31-71. [CrossRef]

23. Harjanto, F.; Wang, Z.; Lu, S.; Tsoi, A.C.; Feng, D.D. Investigating the impact of frame rate towards robust human action recognition. Signal Process. 2016, 124, 220-232. [CrossRef]

24. Lane, N.; Mohammod, M.; Lin, M.; Yang, X.; Lu, H.; Ali, S.; Doryab, A.; Berke, E.; Choudhury, T.; Campbell, A.B. A smartphone application to monitor, model and promote wellbeing. In Proceedings of the 5th International ICST Conference on Pervasive Computing Technologies for Healthcare, Dublin, Ireland, 23-26 May 2011; pp. 23-26.

25. Kose, M.; Incel, O.; Ersoy, C. Online Human Activity Recognition on Smart Phones. In Proceedings of the Workshop on Mobile Sensing: From Smartphones and Wearables to Big Data, Beijing, China, 16-20 April 2012; pp. 11-15.

26. Das, S.; Green, L.; Perez, B.; Murphy, M.; Perring, A. Detecting User Activities Using the Accelerometer on Android Smartphones; Technical Report; Carnegie Mellon University: Pittsburgh, PA, USA, 2010.

27. Thiemjarus, S.; Henpraserttae, A.; Marukatat, S. A study on instance-based learning with reduced training prototypes for device-context-independent activity recognition on a mobile phone. In Proceedings of the 2013 IEEE International Conference on Body Sensor Networks (BSN), Cambridge, MA, USA, 6-9 May 2013; pp. 1-6.

28. Kim, T.; Cho, J.; Kim, J. Mobile Motion Sensor-Based Human Activity Recognition and Energy Expenditure Estimation in Building Environments. Smart Innov. Syst. Technol. 2013, 22, 987-993.

29. Siirtola, P. Recognizing Human Activities User-independently on Smartphones Based on Accelerometer Data. Int. J. Interact. Multimed. Artif. Intell. 2012, 1, 38-45. [CrossRef] 
30. Zhao, K.; Du, J.; Li, C.; Zhang, C.; Liu, H.; Xu, C. Healthy: A Diary System Based on Activity Recognition Using Smartphone. In Proceedings of the 2013 IEEE 10th International Conference on Mobile Ad-Hoc and Sensor Systems (MASS), Hangzhou, China, 14-16 October 2013; pp. 290-294.

31. Khan, A.; Siddiqi, M.; Lee, S. Exploratory Data Analysis of Acceleration Signals to Select Light-Weight and Accurate Features for Real-Time Activity Recognition on Smartphones. Sensor 2013, 13, 13099-13122. [CrossRef] [PubMed]

32. Guiry, J.; van de Ven, P.; Nelson, J. Orientation independent human mobility monitoring with an android smartphone. In Proceedings of the IASTED International Conference on Assistive Technologies, Innsbruck, Austria, 15-17 February 2012; pp. 800-808.

33. San-Segundo-Hernández, R.; Lorenzo-Trueba, J.; Martínez-González, B.; Pardo, J.M. Segmenting human activities based on HMMs using smartphone inertial sensors. Pervasive Mob. Comput. 2016, 30, 84-96. [CrossRef]

34. San-Segundo, R.; Montero, J.M.; Barra-Chicote, R.; Fernández, F.; Pardo, J.M. Feature extraction from smartphone inertial signals for human activity segmentation. Signal Process. 2016, 120, 359-372. [CrossRef]

35. Cho, H.; Yoon, S.M. Divide and Conquer-Based 1D CNN Human Activity Recognition Using Test Data Sharpening. Sensors 2018, 18, 1055.

36. Ignatov, A. Real-time human activity recognition from accelerometer data using Convolutional Neural Networks. Appl. Soft Comput. 2018, 62, 915-922. [CrossRef]

37. Jiang, W.; Yin, Z. Human Activity Recognition Using Wearable Sensors by Deep Convolutional Neural Networks. In Proceedings of the 23rd ACM International Conference on Multimedia (MM'15), Brisbane, Australia, 26-30 October 2015; ACM: New York, NY, USA, 2015; pp. 1307-1310.

38. Ronao, C.A.; Cho, S.B. Human activity recognition with smartphone sensors using deep learning neural networks. Expert Syst. Appl. 2016, 59, 235-244. [CrossRef]

39. Zeng, M.; Nguyen, L.T.; Yu, B.; Mengshoel, O.J.; Zhu, J.; Wu, P.; Zhang, J. Convolutional Neural Networks for human activity recognition using mobile sensors. In Proceedings of the 6th International Conference on Mobile Computing, Applications and Services, Austin, TX, USA, 6-7 November 2014; pp. 197-205.

40. Yang, J.B.; Nguyen, M.N.; San, P.P.; Li, X.L.; Krishnaswamy, S. Deep Convolutional Neural Networks on Multichannel Time Series for Human Activity Recognition. In Proceedings of the 24th International Conference on Artificial Intelligence (IJCAI'15), Buenos Aires, Argentina, 25 July-31 August 2015; AAAI Press: Menlo Park, CA, USA, 2015; pp. 3995-4001.

41. Inoue, M.; Inoue, S.; Nishida, T. Deep Recurrent Neural Network for Mobile Human Activity Recognition with High Throughput. Artif. Life Robot. 2018, 23, 173-185. [CrossRef]

42. Ordóñez, F.J.; Roggen, D. Deep Convolutional and LSTM Recurrent Neural Networks for Multimodal Wearable Activity Recognition. Sensors 2016, 16, 115. [CrossRef] [PubMed]

43. Edel, M.; Köppe, E. Binarized-BLSTM-RNN based Human Activity Recognition. In Proceedings of the 2016 International Conference on Indoor Positioning and Indoor Navigation (IPIN), Madrid, Spain, 4-7 October 2016; pp. 1-7.

44. Kingma, D.P.; Ba, J. Adam: A Method for Stochastic Optimization. arXiv 2014, arXiv:1412.6980,

45. Shakya, S.R.; Zhang, C.; Zhou, Z. Comparative Study of Machine Learning and Deep Learning Architecture for Human Activity Recognition Using Accelerometer Data. Int. J. Mach. Learn. Comput. 2018, 8, 577-582.

(C) 2019 by the authors. Licensee MDPI, Basel, Switzerland. This article is an open access article distributed under the terms and conditions of the Creative Commons Attribution (CC BY) license (http://creativecommons.org/licenses/by/4.0/). 